\title{
The INTREST registry: protocol of a multicenter prospective cohort study of predictors of women's response to integrative breast cancer treatment
}

Heidemarie Haller ${ }^{1 *}$ (D), Petra Voiß ${ }^{1,2}$, Holger Cramer $^{1}$, Anna Paul ${ }^{1}$, Mattea Reinisch², Sebastian Appelbaum³, Gustav Dobos ${ }^{1}$, Georg Sauer ${ }^{4}$, Sherko Kümmel ${ }^{2}$ and Thomas Ostermann ${ }^{3}$

\begin{abstract}
Background: Cancer registries usually assess data of conventional treatments and/or patient survival. Beyond that, little is known about the influence of other predictors of treatment response related to the use of complementary therapies (CM) and lifestyle factors affecting patients' quality and quantity of life.

Methods: INTREST is a prospective cohort study collecting register data at multiple German certified cancer centers, which provide individualized, integrative, in- and outpatient breast cancer care. Patient-reported outcomes and clinical cancer data of anticipated $N=715$ women with pTNM stage I-III breast cancer are collected using standardized case report forms at the time of diagnosis, after completing neo-/adjuvant chemotherapy, after completing adjuvant therapy (with the exception of endocrine therapy) as well as 1, 2, 5, and 10 years after baseline. Endpoints for multivariable prediction models are quality of life, fatigue, treatment adherence, and progression-based outcomes/survival. Predictors include the study center, sociodemographic characteristics, histologic cancer and comorbidity data, performance status, stress perception, depression, anxiety, sleep quality, spirituality, social support, physical activity, diet behavior, type of conventional treatments, use of and belief in CM treatments, and participation in a clinical trial. Safety is recorded following the Common Terminology Criteria for Adverse Events.
\end{abstract}

Discussion: This trial is currently recruiting participants. Future analyses will allow to identify predictors of shortand long-term response to integrative breast cancer treatment in women, which, in turn, may improve cancer care as well as quality and quantity of life with cancer.

Trial registration: German Clinical Trial Register DRKS00014852. Retrospectively registered at July 4th, 2018. Keywords: Breast Cancer, Predictors, Treatment response, Integrative Cancer treatment, Complementary medicine

\footnotetext{
* Correspondence: h.haller@kem-med.com

'Department of Internal and Integrative Medicine, Evang. Kliniken Essen-Mitte, Faculty of Medicine, University of Duisburg-Essen, Am Deimelsberg 34a, 45276 Essen, Germany

Full list of author information is available at the end of the article
}

(C) The Author(s). 2021 Open Access This article is licensed under a Creative Commons Attribution 4.0 International License, which permits use, sharing, adaptation, distribution and reproduction in any medium or format, as long as you give appropriate credit to the original author(s) and the source, provide a link to the Creative Commons licence, and indicate if changes were made. The images or other third party material in this article are included in the article's Creative Commons licence, unless indicated otherwise in a credit line to the material. If material is not included in the article's Creative Commons licence and your intended use is not permitted by statutory regulation or exceeds the permitted use, you will need to obtain permission directly from the copyright holder. To view a copy of this licence, visit http://creativecommons.org/licenses/by/4.0/ The Creative Commons Public Domain Dedication waiver (http://creativecommons.org/publicdomain/zero/1.0/) applies to the data made available in this article, unless otherwise stated in a credit line to the data. 


\section{Background}

With an estimated prevalence of 100.5 million in 2017, cancer remains one of the leading causes of mortality worldwide [1]. For patients diagnosed with breast cancer, the prognosis has continuously improved, resulting in $33.8 \%$ more years lived with disability over the last decade [1]. Thus, aspects of quality of life and management of side effects during and after cancer treatment have progressively increasing impact. Common symptoms associated with the diagnosis and treatment of cancer include fatigue, sleep disturbances, affective disorders, pain and neuropathy, reported by more than half of all patients [2-4]. In order to improve the quality of life, cancer patients often use complementary medicine (CM) [5-8]. On average, up to $40 \%$ of cancer patients use CM across Europe [6], with breast cancer patients being the largest group $[6,9]$. They, however, tend not to disclose their usage of CM treatments to their treating oncologists [6]. In the worst case, this lack of communication can lead to severe interactions between complementary and conventional therapies that can have a negative impact not only on quality but also on quantity of life [10-13].

In contrast, integrative oncology "is a patient-centered, evidence-informed field of cancer care that utilizes mind and body practices, natural products, and/or lifestyle modifications from different traditions alongside conventional cancer treatments. Integrative oncology aims to optimize health, quality of life, and clinical outcomes across the cancer care continuum and to empower people to prevent cancer and become active participants before, during, and beyond cancer treatment" [14]. The concept is based on the NIH definition of integrative health care [15] and Clinical Practice Guidelines of the Society for Integrative Oncology (SIO) [16], endorsed by the American Society of Clinical Oncology (ASCO) [17]. A systematic review published in 2012 identified 29 integrative cancer programs, situated in the United States, England, Canada, and Germany [18], all of them established between 1968 and 2007. In 2010, in Germany another program was launched that provides integrative oncology for breast cancer patients by incorporating evidence-based complementary therapies into standard in- and outpatient cancer care [19]. Beside conventional curative and adjuvant cancer treatments and supportive psycho-oncological care, the individualized CM treatments offered in this program include:

CM treatments administrated by physicians/naturopaths/ acupuncturists such as

- individual and group-based educative consultations about the benefits and risks of CM therapies in the management of side effects of conventional cancer treatment,
- natural products such as herbs, dietary supplements, mistletoe, and other anthroposophical/homeopathic remedies,

- ear and body acupuncture and acupressure, neural therapy, gua sha therapy (traditional Chinese skin scraping massage), and cupping,

CM treatments administrated by mind-body medicine therapists such as

- individual and group-based educative consultations about the benefits and risks of lifestyle modifications in the areas of exercise, nutrition, relaxation, cognitive restructuring, and naturopathic coping strategies,

- courses in yoga, tai chi and qi gong, art therapy, mindfulness, relaxation, and meditation,

CM treatments administrated by nurses such as

- aromatherapy with and without (rhythmical) massage, therapeutic foot massage, footbaths, compresses and poultices, teas and mouthwashes,

- psychosocial support groups [19-21].

To address requested CM therapies with currently insufficient scientific evidence, a traffic light system has been developed [21]. Therapies are given a green light and can be used if they are of low risk and are known to have valuable clinical effects. Promising therapies that are of higher risk as well as those that have shown a limited extent of efficacy while having only low risks are given a yellow light and might be used in selected cases. Therapies with limited effectiveness and high risks shall not be applied (red light).

Randomized controlled trials have shown preliminary comparative effectiveness of integrative cancer programs in contrast to treatment as usual. One trial investigated outpatients with breast and gynecologic cancer undergoing chemotherapy and found that an integrative complex nursing intervention over 24 weeks customized to the patients' symptomatic burden and preferences improved aspects of quality of life and fatigue [22]. Another trial examined breast cancer patients in different treatment phases who were randomized to either standard care alone or standard care integrating individualized CM treatments provided by trained physicians and nurses during a period of 26 weeks. Analyses revealed significant between-group differences on cancer-related quality of life, pain, fatigue, and function in favor of the integrative therapy [23]. However, RCT designs often do not include follow-ups longer than 6 or 12 months, which limits their external validity. Clinical and epidemiological cancer registries, on the other hand, follow patients 
without time restrictions but usually do not go further than assessing data on conventional treatment algorithms and patient survival.

Predictors of response to cancer treatment/patient survival include, in particular, tumor characteristics and access to innovative, timely screening and treatment strategies [24-27]. Moreover, factors of resilience related to mental, physical and social variables, such as the patient's ability to adapt to psychological distress or stressful life events without developing mental health disorders [28, 29], exercise and healthy diet [30-32], are shown to be predictors of survival from breast cancer. A registry assessing data on the influence of both conventional and CM treatment and of mental and physical resilience using qualitative and quantitative variables of treatment response has not been established yet.

The INTREST registry aims to identify independent predictors of treatment-response in women undergoing individualized, integrative breast cancer treatment combining conventional as well as supportive complementary therapies. Treatment response is defined in terms of quality of life, fatigue, treatment adherence to conventional cancer care, and progression-based variables.

\section{Methods}

\section{Study setting and design}

We developed the INTREST registry as an epidemiological, prospective, multi-center cohort design according to the STROBE and TRIPOD guidelines [33, 34]. The study protocol was reported according to the SPIRIT guideline [35]. Each study site was approved by the respective ethics committee and registered at the WHO International Clinical Trials Registry Platform/German Clinical Trials Register (DRKS00014852). The 2017 version of the INTREST protocol comprised two certified cancer centers in Germany, one in Essen (Breast Unit, Evang. Kliniken Essen-Mitte) and a second in Stuttgart (Department of Gynecology, Robert-Bosch-Hospital). The 2019 version includes a third center located in Münster, Germany (Breast Unit, St. Franziskus-Hospital). Involving further study sites is within the scope of the protocol.

Qualified study sites have to be certified cancer centers providing conventional breast cancer diagnosis and treatment procedures as well as integrate in- and/or outpatient complementary medical visits, treatment offers, and nursing interventions [19, 21]. Women shall be treated by an interdisciplinary medical team of breast cancer specialists (radiologists, pathologists, breast surgeons, and oncologists), complementary physicians, psycho-oncologists, mind-body therapists, or breast care nurses trained in complementary medicine. CM treatments are offered in accordance with the individual indication and preference during and after active cancer therapy up to 10 years post diagnosis.
Measurement points include a baseline assessment at the time of diagnosis (T0), two individual time points after completing the neo-/adjuvant chemotherapy (T1) and/or after finishing all adjuvant treatments (except for endocrine therapy) (T2), a one-year follow-up (T3) as well as further consultations at year two (T4), year five (T5), and year ten (T6). In cases of withdrawal of consent or death, study participation ends earlier. A summary of the study design can be found in Fig. 1.

\section{Eligibility criteria}

Patients are eligible, if they are female, have been diagnosed with primary breast cancer stage I-III (according to the pTNM classification) [36], and receive individualized cancer treatments in one of the participating study centers. Breast cancer diagnosis has to be confirmed by histological classification following breast biopsy according to the currently applicable guidelines [37, 38]. Exclusion criteria are male sex, hospitalization due to treatment of a benign tumor, carcinoma in situ, relapse or metastases. Women who report suffering from a severe comorbid somatic disease or being under psychopharmacological treatment for a psychiatric or neurodegenerative disorder, such as dementia, major depression or a psychosis that make them unable to participate in the study are excluded, as well as those who do not have the sufficient knowledge of the German language.

\section{Recruiting procedure}

Eligible women are selected from tumor board lists of the participating hospitals that contain the latest on-site histologic, genetic, and clinical diagnoses as well as treatment recommendations. Study nurses inform eligible patients about the study procedure and provide written study information as part of the tumor board outpatient visit, where women receive their individualized treatment plans. In order to be included in the study, interested women have to give their written informed consent and fill out the baseline questionnaire. Reasons for non-participation are recorded.

Follow-up questionnaires are delivered by mail at predefined time points and are monitored by reminder calls. Replacement questionnaires are provided when necessary [39]. In cases of no response, additional questionnaires are sent via email. Considering the 10-year follow-up period and possible mental deterioration, a screening tool for cognitive impairment is included. The Mail-In Cognitive Function Screening Instrument (MCFSI) is assessed at T5 and T6 [40]. In cases of $\geq 5$ points on the MCFSI [41], respective questionnaires are checked for response bias and excluded from analyses/ further monitoring if necessary. In general, study staff is instructed to verify survival status of each woman by 


\begin{tabular}{|c|c|c|c|c|c|c|c|c|c|}
\hline & & \multicolumn{8}{|c|}{ STUDY PERIOD } \\
\hline & & \multirow[b]{2}{*}{ Enrollment } & \multirow[b]{2}{*}{$\begin{array}{c}\text { Active cancer } \\
\text { treatment }\end{array}$} & & & \multicolumn{4}{|c|}{ Follow-up } \\
\hline & & & & \multicolumn{2}{|c|}{ Post intervention } & 1 Year & 2 Years & 5 Years & 10 Years \\
\hline & TIME POINT & $T_{0}$ & & $T_{1}$ & $T_{2}$ & $T_{3}$ & $T_{4}$ & $T_{5}$ & $T_{6}$ \\
\hline \multirow{3}{*}{ 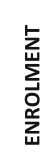 } & Eligibility screening & $\mathrm{x}$ & & & & & & & \\
\hline & Enrollment visit & $x$ & & & & & & & \\
\hline & Informed consent & $\mathrm{x}$ & & & & & & & \\
\hline \multirow{2}{*}{ 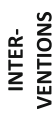 } & $\begin{array}{r}\text { Inpatient integrative } \\
\text { cancer treatment }\end{array}$ & & $x$ & & & & & & \\
\hline & $\begin{array}{r}\text { Outpatient integrative } \\
\text { cancer treatment }\end{array}$ & & $x$ & $\mathrm{x}$ & $x$ & $\mathrm{x}$ & $x$ & $x$ & $x$ \\
\hline \multirow{6}{*}{ 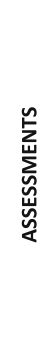 } & Social demographics & $x$ & & & & & & & \\
\hline & Medical records & $\mathrm{x}$ & & & & $\mathrm{x}$ & $\mathrm{x}$ & $\mathrm{x}$ & $\mathrm{x}$ \\
\hline & $\begin{array}{l}\text { Baseline data of } \\
\text { response variables }\end{array}$ & $\mathrm{x}$ & & & & & & & \\
\hline & Response variables & & & $x$ & $x$ & $x$ & $\mathrm{x}$ & $\mathrm{x}$ & $\mathrm{x}$ \\
\hline & Predictor variables & $x$ & & $\mathrm{x}$ & $\mathrm{x}$ & $\mathrm{x}$ & $\mathrm{x}$ & $\mathrm{x}$ & \\
\hline & Adverse events & & & $x$ & $x$ & $x$ & $\mathrm{x}$ & $\mathrm{x}$ & $x$ \\
\hline
\end{tabular}

Fig. 1 Study flow diagram

checking the internal cancer registry before sending the respective questionnaire.

\section{Data collection}

\section{Outcomes of treatment response}

Endpoints for the multivariate prediction model include patient reported outcomes (PROs), treatment adherence, and progression-based outcomes.

PROs include quality of life and fatigue, which are assessed by the Functional Assessment of Cancer Therapy General (FACT-G) [42] and the associated Fatigue Scale (FACIT-F) [43]. The FACT-G total score ranges from 0 to 108 points. The highest possible score for the physical, social, and functional well-being sub scales is 28 , and 24 for the emotional well-being sub scale. The FACIT-F ranges from 0 to 52 points. Both questionnaires have been well validated and have shown good reliability $[44,45]$ as well as a better responsiveness for the detection of clinically relevant changes compared to the FACT-B [46] or the EORTC QLQ-C30 [47].

Treatment responders at the time points $\mathrm{T} 1$ to $\mathrm{T} 2$ are defined as women who will score above the 25 th percentile of the female US adult population ( $>68$ points on the FACT-G and $>33$ points on the FACIT-F) [44] and will reach the same or higher scores in comparison to their respective baseline values at T0. Non-responder are defined as women who will score below the respective 25th percentile of the female US adult population or will report a minimal clinically important worsening (7 points on the FACT-G total score or 4 points on the FACIT-F) $[45,48]$ in comparison to their respective baseline values at T0. Responders at the time points T3 to T6 are defined as women who will score above the 50th percentile of the female US adult population $(>83$ points on the FACT-G and $>42$ points on the FACIT-F) [44] and will reach a minimal clinically important improvement ( $>5$ points on the FACT-G total score or 4 points on the FACIT-F) $[44,45]$ in comparison to their respective baseline values at $\mathrm{T} 0$. Non-responders are defined as women who will score below the respective 50th percentile or will report a minimal clinically important worsening.

Adherence to chemotherapy and endocrine therapy is assessed at T1 and T4, T5, and T6, respectively. Patients who do not adhere to chemo- or endocrine therapy due to adverse events are defined as non-responders, others as responders.

Progression-based outcomes recommended for the adjuvant setting [49] include Invasive Disease-Free Survival (IDFS), Distant Relapse-free Survival (DRFS), Breast Cancer Specific Survival (BCSS) and Overall Survival $(\mathrm{OV})$. Treatment response and non-response for all progression-based outcomes is assessed at T3 to T6.

\section{Predictors}

Predictor variables include sociodemographic characteristics at T0 such as age, race, marital status, kind of health insurance, education, occupation, height and 
weight, lifestyle habits (alcohol intake, smoking behavior, physical activity, and diet), characteristics of menstruation, pregnancy and the use of hormonal contraception, other hormonal drugs for in vitro fertilization, abortion or menopausal symptoms, and radiation exposure. Patients are also questioned about their beliefs about CM therapies using the 10-item CAM Health Belief Questionnaire (CHBQ) [50], and about their prior use of and interest in $\mathrm{CM}$ using a 10-point numeric rating scales (NRS).

Further clinical data at T0 are obtained from medical records. Those include time of diagnosis, histology, previous oncological disease, hereditary risk score, pTNM and if indicated ypTNM, grading, hormone receptor status, menopausal status, ECOG and Karnofsky performance status, and physical and mental comorbidity according to the 35-item Health-Related Quality of Life Comorbidity Index (HRQL-CI) [51].

Predictors assessed by questionnaires at all time points include stress perception using the 10-item Questionnaire on Distress in Cancer Patients Short Form (QSC-R10) [52], depression using the 10-item Center for Epidemiologic Studies Depression Scale Short Form (CESD-SF10) $[53,54]$, hopelessness using the negatively worded 2-item Brief Hopelessness measure (BH-NEG) [55], state anxiety using the Patient-Reported Outcomes Measurement Information System Emotional Distress Anxiety 4-item Short Form (PROMIS-EDA-SF4) [56], progression anxiety using the 5-item Fear of Relapse/Recurrence Scale (FRRS) [57], emotion regulation using the 4-item expressive suppression subscale of the Emotion Regulation Questionnaire (ERQ) $[58,59]$, sleep disturbance using the PatientReported Outcomes Measurement Information System Sleep Disturbance 4-item Short Form (PROMIS-SD-SF4) [60], spiritual well-being using the 12-item Functional Assessment of Cancer Therapy Spiritual Well-Being Scale (FACIT-SP) [61], social support using the 8-item perceived available support subscales of the Berlin Social Support Scales (BSSS) [62], physical activity using the 4item International Physical Activity Questionnaire (IPAQ) [63], and diet using the 14-item Mediterranean Diet Adherence Screener (MEDAS) [64].

Finally, we assess variables of the study center and the need for and type of conventional treatment procedures including data on the surgical intervention, chemotherapy, radiotherapy, and endocrine therapy as well as whether or not the patient is participating in a clinical trial. At all follow-ups, the patients are asked about their use of complementary therapies (provided at the study center as well as used by patients themselves) by an extended version (59 predefined and additional free-text items instead of originally 29 ones) of the International Complementary and Alternative Medicine Questionnaire (I-CAM-Q) [65-67]. Adverse events are recorded by the 32-item Short Form of the Memorial Symptom Assessment Scale (MSAS-SF) [68] following the Common Terminology Criteria for Adverse Events. Satisfaction with the treatments is assessed by the 8-item Client Satisfaction Questionnaire (CSQ) [69].

\section{Data management and quality assurance}

All patient-reported data and those collected from medical records are transferred into standardized paper CRFs by trained medical staff of the respective study site. After formal checking for completeness, data from the paper CRFs are entered into a specially designed online database based on WINDOWs package XAMPP including an Apache Server, a MySQL-Database, and PHP as the dialect of the framework [70].

The INTREST-database uses a web-application, which serves as a secured user interface to access the database. Entered data is validated during the data entry process using programmed validation checks concerning item types, checks for required values and item ranges. For each discrepancy, a "discrepancy note" is stored in the system and resolved by cross-checking the entries in the database with the source data. Discrepancies that cannot be solved, are indicated as "unsolved" in the system and will be discussed at a regular data review meeting.

The INTREST-registry is provided to each study site and administrated centrally by the evaluating body (Prof. Dr. Thomas Ostermann, University of Witten / Herdecke). Transfer of registry data takes place exclusively in a pseudonymized form by the study centers. Thus, the conclusion on an individual participant is not possible. For statistical analysis, registry data are transferred into a predefined csvfile format. Also at this stage, data are examined for accuracy and completeness by random comparisons between the csv-file and the original database.

\section{Statistics \\ Sample size estimation}

Taking into account $\mathrm{k}$ independent variables as covariates and a defined ratio $\mathrm{p}$ of responders to non-responders at the respective follow-up points, a required number of cases of $N=10 \mathrm{k} / \mathrm{p}$ are needed [71]. To avoid overfitting and in line with the recommendations given by Vittinghoff \& McCulloch, 2007 [72] we assume to arrive at ten predictors out of the above mentioned variables. Together with a ratio $\mathrm{p}$ of $1: 5$ of responders to non-responders this results in a sample size of $N=10 \times 10 / 0.20=500$. To compensate for power losses due to drop-outs of up to $30 \%$, for T0, a sample size of $N=715$ is calculated.

\section{Dealing with missing data}

In responder analysis, there are two strategies to deal with missing data: imputation of missing data prior to dichotomization or dichotomization prior to imputation. 
In simulation studies, imputing data before dichotomization was shown to be less biased compared with imputing the dichotomous response [73]. Therefore, data missing at random will be imputed i.e. by using Markov chain Monte Carlo methods.

\section{Statistical analysis plan}

The method to be used for predicting responders and non-responders will be based on imputation strategies for responder analysis given in the model selection approach by Schomaker \& Heumann, 2014 [74]. In practical terms, we follow the outline given in Rethorst et al., 2017 [75]. The single steps in our approach are displayed in the supplementary material and described as follows:

From the original set of data, a set of bootstrap samples $B_{i}(i=1 \ldots m)$ is drawn. Missing values in each bootstrap sample are replaced by multiple imputation techniques described above resulting in $k$ completed data $B_{i j}(i=1 \ldots m ; j=1, k)$ sets for each bootstrap sample. For each of the data sets $B_{i j}$ a separate model selection approach is carried out using two different methods.

1. A logistic regression model using a least absolute shrinkage and selection operator (LASSO) approach [76]: Although similar to the classical logistic regression model, the LASSO regression model has an additional penalty term $\lambda>0$, which shrinks coefficients of predictors with low predictive power towards zero or exactly to zero [77].

2. A random forest (RF) classification method: the random forest is a classification method based on the binary classification and regression trees (CART) method in data mining, first introduced by Breiman, 2001 [78]. Based on $\mathrm{k}$ learning sets, the $\mathrm{K}$ decision trees form a random forest. Then, the majority vote of these trees is used to make an ensemble classification decision [79].

Resulting predictors $\mathrm{P}_{i j l}$ of these modeling approaches will be pooled and the $\mathrm{k}$ estimates for each bootstrap sample $B_{i}$ will be averaged. Based on these results, a final model for each selection approach will be created taking into account the rank and relative strength of the predictors from each bootstrap sample.

The final two models (one for the LASSO and one for the RF approach) are used for a) a classical logistic regression model and b) a Classification and Regression Trees (CART) approach. In this final evaluation step, a validation of the predictors is performed using the compete cases of the out of back samples $\mathrm{O}_{\mathrm{i}}(i=1 \ldots m)$ left over from the bootstrap datasets. All statistical analyses are going to be run with $\mathrm{R}$.

\section{Discussion}

The analysis of the INTREST registry data will reveal predictors of response to integrative breast cancer treatment. Challenges of the study design may include the overall large number of questionnaires as well as the 10year follow-up, which may result in a high number of drop-outs. To improve patient initial participation and attendance, only significant predictors will remain within the revised INTREST-registry, while non-significant variables/questionnaires will be removed. In addition, comprehensive monitoring strategies shall ensure higher patient compliance.

For other cancer centers providing integrative cancer care, the adapted INTREST-registry may provide an interesting platform to answer questions of health services research. Selected significant predictors might also be of interest for standard clinical cancer registries to extend their validity and reliability. Particularly, if the use of $\mathrm{CM}$ therapies proves to be a significant predictor, those with adequate evidence of effectiveness and safety may be suggested to be integrated into standard cancer care. This will serve the needs of the women diagnosed with breast cancer $[8,9,80]$ and may improve quality of care for cancer as well as quality and quantity of life with cancer.

\section{Trial status}

The trial is currently recruiting participants.

\begin{abstract}
Abbreviations
ASCO: American Society of Clinical Oncology; BCSS: Breast Cancer Specific Survival; CAM: Complementary and Alternative Medicine; CART: Classification and Regression Trees; CM: Complementary Medicine; CRF: Case Report Form; CSF: Comma-separated values (Excel) file; DRFS: Distant Relapse-free Survival; ECOG: Eastern Cooperative Oncology Group performance status;

IDFS: Invasive Disease-Free Survival; INTREST: RESposne to INTegrative breast cancer Treatment; $\wedge$ : Lambda; LASSO: Least absolute shrinkage and selection operator; MCFSI: Mail-In Cognitive Function Screening Instrument; MYSQ L: Open-source relational database management system; OV: Overall Survival; PHP: Programming language; PRO: Patient Reported Outcome; pTNM: Classification of Malignant Tumors, assessed after surgery; R: Free programming language software; RF: Random Forest; SIO: Society for Integrative Oncology; STROBE: STrengthening the Reporting of OBservational studies in Epidemiology guideline; T: Time point of data assessment; TRIPOD: Transparent Reporting of a multivariable prediction model for Individual Prognosis Or Diagnosis guideline; XAMPP: Open-source web server solution package by Apache Friends; yTNM: Classification of Malignant Tumors, assessed after neoadjuvant chemotherapy and/or radiation therapy
\end{abstract}

\section{Supplementary Information}

The online version contains supplementary material available at https://doi. org/10.1186/s12885-021-08468-2.

Additional file 1. Statistical analysis plan. 


\section{Authors' contributions}

$\mathrm{HH}$ and TO were responsible for the conception and design of the study and for drafting the manuscript. AP, GD, GS, HC, MR, PV, SA, and SK participated in the conception and design of the study and critically revised the manuscript. All authors approved the final manuscript.

\section{Funding}

The study was supported by a research grant from the Robert-Bosch Foundation, Postfach 501120, 70341 Stuttgart, Germany. The funding source had no influence on the design of the study or the preparation or approval of this manuscript.

\section{Availability of data and materials}

The dataset analyzed during the study is not publicly available as it include personal/pseudonymized information. Fully anonymized data will be available from the corresponding author on reasonable request.

\section{Declarations}

\section{Ethics approval and consent to participate}

The study protocol was developed in accordance with the Declaration of Helsinki and approved by the Ethics Committee of the Ärztekammer Nordrhein (approval number: 2017381), Ethics Committee of the Landesärztekammer Baden-Wuerttemberg (approval number: B-F-2017-009), and by the Ethics Committee of the Ärztekammer Westfalen-Lippe (approval number: 2019-503-b-S) for the respective study centers. All participants have to give their written informed consent for the storage and analysis of their pseudonymized data before they were included in the study.

\section{Consent for publication}

All participants have to give their written informed consent for the publication of their anonymized data.

\section{Competing interests}

The authors declare that they have no competing interests.

\section{Author details}

${ }^{1}$ Department of Internal and Integrative Medicine, Evang. Kliniken Essen-Mitte, Faculty of Medicine, University of Duisburg-Essen, Am Deimelsberg 34a, 45276 Essen, Germany. ${ }^{2}$ Breast Unit, Evang. Kliniken Essen-Mitte, Essen, Germany. ${ }^{3}$ Department of Psychology, Chair of Research Methodology and Statistics in Psychology, Witten / Herdecke University, Witten, Germany. ${ }^{4}$ Department of Gynecology and Obstetrics, Robert-Bosch-Hospital, Stuttgart, Germany.

Received: 30 April 2020 Accepted: 9 June 2021

Published online: 23 June 2021

\section{References}

1. GBD 2017 Disease and Injury Incidence and Prevalence Collaborators. Global, regional, and national incidence, prevalence, and years lived with disability for 354 diseases and injuries for 195 countries and territories, 1990-2017: a systematic analysis for the global burden of disease study 2017. Lancet. 2018;392(10159):1789-858.

2. Cheng KK, Yeung RM. Impact of mood disturbance, sleep disturbance, fatigue and pain among patients receiving cancer therapy. Eur J Cancer Care (Engl). 2013;22(1):70-8. https:/doi.org/10.1111/j.1365-2354.2012.01372.x.

3. Patrick DL, Ferketich SL, Frame PS, Harris JJ, Hendricks CB, Levin B, et al. National Institutes of Health state-of-the-science conference statement: symptom management in cancer: pain, depression, and fatigue, July 15-17. J Natl Cancer Inst Monogr. 2002;2004(32):9-16.

4. Singer S, Das-Munshi J, Brahler E. Prevalence of mental health conditions in cancer patients in acute care--a meta-analysis. Ann Oncol. 2010;21(5):92530. https://doi.org/10.1093/annonc/mdp515.

5. McLay JS, Stewart D, George J, Rore C, Heys SD. Complementary and alternative medicines use by Scottish women with breast cancer. What, why and the potential for drug interactions? Eur J Clin Pharmacol. 2012;68(5): 811-9. https://doi.org/10.1007/s00228-011-1181-6.

6. Molassiotis A, Fernadez-Ortega P, Pud D, Ozden G, Scott JA, Panteli V, et al. Use of complementary and alternative medicine in cancer patients: a
European survey. Ann Oncol. 2005;16(4):655-63. https://doi.org/10.1093/a nnonc/mdi110.

7. Sewitch MJ, Yaffe M, Maisonneuve J, Prchal J, Ciampi A. Use of complementary and alternative medicine by cancer patients at a Montreal hospital. Integrative cancer therapies. 2011;10(4):305-11. https://doi.org/1 $0.1177 / 1534735410395136$

8. Verhoef MJ, Balneaves LG, Boon HS, Vroegindewey A. Reasons for and characteristics associated with complementary and alternative medicine use among adult cancer patients: a systematic review. Integrative cancer therapies. 2005;4(4):274-86. https://doi.org/10.1177/1534735405282361.

9. Boon HS, Olatunde F, Zick SM. Trends in complementary/alternative medicine use by breast cancer survivors: comparing survey data from 1998 and 2005. BMC Womens Health. 2007;7(1):4. https://doi.org/10.1186/14726874-7-4.

10. Alsanad SM, Williamson EM, Howard RL. Cancer patients at risk of herb/food supplement-drug interactions: a systematic review. Phytotherapy research : PTR. 2014;28(12):1749-55. https://doi.org/10.1002/ptr.5213.

11. Ben-Arye E, Samuels N, Goldstein LH, Mutafoglu K, Omran S, Schiff E, et al. Potential risks associated with traditional herbal medicine use in cancer care: a study of MiddleEastern oncology health care professionals. Cancer. 2016;122(4):598-610. https://doi.org/10.1002/cncr.2 9796.

12. Bode AM, Dong Z. Toxic phytochemicals and their potential risks for human cancer. Cancer Prevent Res (Philadelphia, Pa). 2015;8(1):1-8.

13. Zeller T, Muenstedt K, Stoll C, Schweder J, Senf B, Ruckhaeberle E, et al. Potential interactions of complementary and alternative medicine with cancer therapy in outpatients with gynecological cancer in a comprehensive cancer center. J Cancer Res Clin Oncol. 2013;139(3):357-65. https://doi.org/10.1007/s00432-012-1336-6.

14. Witt CM, Balneaves LG, Cardoso MJ, Cohen L, Greenlee H, Johnstone P, Kucuk O, Mailman J, Mao JJ. A Comprehensive Definition for Integrative Oncology. J Natl Cancer Inst Monogr. 2017;2017(52):Igx012. https://doi.org/1 0.1093/jncimonographs/lgx012

15. Complementary, Alternative, or Integrative Health: What's In a Name? [https://nccih.nih.gov/health/integrative-health].

16. Greenlee H, Balneaves LG, Carlson LE, Cohen M, Deng G, Hershman D, et al. Clinical practice guidelines on the use of integrative therapies as supportive care in patients treated for breast cancer. J Natl Cancer Inst Monogr. 2014; 2014(50):346-58. https://doi.org/10.1093/jncimonographs/lgu041.

17. Lyman GH, Greenlee H, Bohlke K, Bao T, DeMichele AM, Deng GE, et al. Integrative therapies during and after breast Cancer treatment: ASCO endorsement of the SIO clinical practice guideline. J Clin Oncol. 2018;36(25): 2647-55. https://doi.org/10.1200/JCO.2018.79.2721.

18. Seely DM, Weeks $L C$, Young S. A systematic review of integrative oncology programs. Curr Oncol. 2012;19(6):e436-61. https://doi.org/10.3747/co.19.1182.

19. Cramer H, Cohen L, Dobos G, Witt CM. Integrative oncology: best of both worlds-theoretical, practical, and research issues. eCAM. 2013;2013:383142.

20. Paul A, Cramer H, Lauche R, Altner N, Langhorst J, Dobos GJ. An oncology mind-body medicine day care clinic: concept and case presentation. Integr Cancer Ther. 2013;12(6):503-7. https://doi.org/10.1177/1534735412473639.

21. Dobos GJ, Voiss P, Schwidde I, Choi KE, Paul A, Kirschbaum B, et al. Integrative oncology for breast cancer patients: introduction of an expertbased model. BMC Cancer. 2012;12(1):539. https://doi.org/10.1186/1471-24 07-12-539.

22. Klafke N, Mahler C, von Hagens C, Uhlmann L, Bentner M, Schneeweiss A, et al. The effects of an integrated supportive care intervention on quality of life outcomes in outpatients with breast and gynecologic cancer undergoing chemotherapy: results from a randomized controlled trial. Cancer Med. 2019;8(8):3666-76. https://doi.org/10.1002/cam4.2196.

23. Witt CM, Ausserer O, Baier S, Heidegger H, Icke K, Mayr O, et al. Effectiveness of an additional individualized multi-component complementary medicine treatment on health-related quality of life in breast cancer patients: a pragmatic randomized trial. Breast Cancer Res Treat. 2015;149(2):449-60. https://doi.org/10.1007/s10549-014-3249-3.

24. Abdel-Rahman O. Incidence and predictors of 1-year mortality among 9236 breast cancer patients referred for adjuvant chemotherapy. Future Oncol (London, England). 2018;14(23):2335-41.

25. Chow CJ, Habermann EB, Abraham A, Zhu Y, Vickers SM, Rothenberger DA, et al. Does enrollment in cancer trials improve survival? J Am Coll Surg. 2013;216(4):774-80; discussion 780-771. https://doi.org/10.1016/j.ja mcollsurg.2012.12.036. 
26. Hunt BR, Silva A, Lock D, Hurlbert M. Predictors of breast cancer mortality among white and black women in large United States cities: an ecologic study. Cancer Causes Control. 2019;30(2):149-64. https://doi.org/10.1007/s1 0552-018-1125-x.

27. Nelson HD, Fu R, Cantor A, Pappas M, Daeges M, Humphrey L. Effectiveness of Breast Cancer Screening: Systematic Review and Meta-analysis to Update the 2009 U.S. preventive services task force recommendation. Ann Intern Med. 2016;164(4):244-55.

28. Chida Y, Hamer M, Wardle J, Steptoe A. Do stress-related psychosocial factors contribute to cancer incidence and survival? Nat Clin Pract Oncol. 2008;5(8):466-75. https://doi.org/10.1038/ncponc1134.

29. Suppli NP, Johansen C, Kessing LV, Toender A, Kroman N, Ewertz M, et al. Survival after early-stage breast Cancer of women previously treated for depression: a Nationwide Danish cohort study. J Clin Oncol : official journal of the American Society of Clinical Oncology. 2017;35(3):334-42. https://doi. org/10.1200/JCO.2016.68.8358.

30. Lahart IM, Metsios GS, Nevill AM, Carmichael AR. Physical activity for women with breast cancer after adjuvant therapy. Cochrane Database Syst Rev. 2018;1:CD011292.

31. Schwingshackl L, Schwedhelm C, Galbete C, Hoffmann G. Adherence to Mediterranean Diet and Risk of Cancer: An Updated Systematic Review and Meta-Analysis. Nutrients. 2017;9(10):1063. https://doi.org/10.3390/nu9101063.

32. Hou R, Wei J, Hu Y, Zhang X, Sun X, Chandrasekar EK, et al. Healthy dietary patterns and risk and survival of breast cancer: a meta-analysis of cohort studies. Cancer Causes Control. 2019;30(8):835-46. https://doi.org/10.1007/s1 0552-019-01193-z.

33. Collins GS, Reitsma JB, Altman DG, Moons KG. Transparent reporting of a multivariable prediction model for individual prognosis or diagnosis (TRIPOD): the TRIPOD statement. Bmj. 2015;350(jan07 4):g7594. https://doi. org/10.1136/bmj.g7594.

34. von Elm E, Altman DG, Egger M, Pocock SJ, Gotzsche PC, Vandenbroucke $J$, et al. Strengthening the reporting of observational studies in epidemiology (STROBE) statement: guidelines for reporting observational studies. Bmj. 2007;335(7624):806-8. https://doi.org/10.1136/bmj.39335.541 782.AD

35. Chan A-W, Tetzlaff JM, Altman DG, Laupacis A, Gøtzsche PC, Krleža-Jerić K, et al. SPIRIT 2013 statement: defining standard protocol items for clinical trials. Ann Intern Med. 2013;158(3):200-7. https://doi.org/10.7326/0003-481 9-158-3-201302050-00583.

36. Brierley JD, Gospodarowicz MK, Wittekind C. TNM classification of malignant Tumours. Oxford: Wiley; 2017

37. NICE. The National Institute for Health and Care Excellence: Advanced Breast Cancer: Diagnosis and Treatment [addendum 2017]. In.; 2009.

38. Deutsche Krebsgesellschaft, Deutsche Krebshilfe, AWMF: [S3-Leitlinie Früherkennung, Diagnose, Therapie und Nachsorge des Mammakarzinoms, Version 4.0, AWMF Registernummer: 032-045OL]. In.; 2017.

39. Hoddinott SN, Bass MJ. The dillman total design survey method. Canadian family physician Medecin de famille canadien. 1986;32:2366-8.

40. Walsh SP, Raman R, Jones KB, Aisen PS. ADCS prevention instrument project: the mail-in cognitive function screening instrument (MCFSI). Alzheimer Dis Assoc Disord. 2006;20(4 Suppl 3):S170-8. https://doi.org/10.1 097/01.wad.0000213879.55547.57.

41. Bade BC, Strange C, Lal C. Effect of obstructive sleep apnea treatment on mail-in cognitive function screening instrument. Am J Med Sci. 2014;348(3): 215-8. https://doi.org/10.1097/MAJ.0000000000000233.

42. Winstead-Fry P, Schultz A. Psychometric analysis of the functional assessment of Cancer therapy-general (FACT-G) scale in a rural sample. Cancer. 1997;79(12):2446-52. https://doi.org/10.1002/(SICI)1097-0142 (19970615)79:12<2446::AID-CNCR23>3.0.CO;2-Q.

43. Yellen SB, Cella DF, Webster K, Blendowski C, Kaplan E. Measuring fatigue and other anemia-related symptoms with the functional assessment of Cancer therapy (FACT) measurement system. J Pain Symptom Manag. 1997; 13(2):63-74. https://doi.org/10.1016/S0885-3924(96)00274-6

44. Brucker PS, Yost K, Cashy J, Webster K, Cella D. General population and cancer patient norms for the functional assessment of Cancer therapygeneral (FACT-G). Evaluation \& the health professions. 2005;28(2):192-211. https://doi.org/10.1177/0163278705275341.

45. Yost KJ, Eton DT. Combining distribution- and anchor-based approaches to determine minimally important differences: the FACIT experience. Evaluation \& the health professions. 2005;28(2):172-91. https://doi.org/10.11 77/0163278705275340.
46. Eton DT, Cella D, Yost KJ, Yount SE, Peterman AH, Neuberg DS, et al. A combination of distribution- and anchor-based approaches determined minimally important differences (MIDs) for four endpoints in a breast cancer scale. J Clin Epidemiol. 2004;57(9):898-910. https://doi.org/10.1016/j.jclinepi.2 004.01.012.

47. King MT, Bell ML, Costa D, Butow P, Oh B. The quality of life questionnaire Core 30 (QLQ-C30) and functional assessment of Cancer-general (FACT-G) differ in responsiveness, relative efficiency, and therefore required sample size. J Clin Epidemiol. 2014;67(1):100-7. https://doi.org/10.1016/j.jclinepi.2 013.02.019.

48. Cella D, Hahn EA, Dineen K. Meaningful change in cancer-specific quality of life scores: differences between improvement and worsening. Quality of life research : an international journal of quality of life aspects of treatment, care and rehabilitation. 2002;11(3):207-21. https://doi.org/10.1023/A:1015276414526.

49. Hudis CA, Barlow WE, Costantino JP, Gray RJ, Pritchard Kl, Chapman JA, et al. Proposal for standardized definitions for efficacy end points in adjuvant breast cancer trials: the STEEP system. J Clin Oncol. 2007;25(15):2127-32. https://doi.org/10.1200/JCO.2006.10.3523.

50. Lie D, Boker J. Development and validation of the CAM health belief questionnaire $(\mathrm{CHBQ})$ and $\mathrm{CAM}$ use and attitudes amongst medical students. BMC Med Educ. 2004;4(1):1-9.

51. Mukherjee B, Ou HT, Wang F, Erickson SR. A new comorbidity index: the health-related quality of life comorbidity index. J Clin Epidemiol. 2011;64(3): 309-19. https://doi.org/10.1016/j.jclinepi.2010.01.025.

52. Book K, Marten-Mittag B, Henrich G, Dinkel A, Scheddel P, Sehlen S, et al. Distress screening in oncology-evaluation of the questionnaire on distress in Cancer patients-short form (QSC-R10) in a German sample. Psychooncology. 2011;20(3):287-93. https://doi.org/10.1002/pon.1821.

53. Andresen EM, Malmgren JA, Carter WB, Patrick DL. Screening for depression in well older adults: evaluation of a short form of the CES-D (Center for Epidemiologic Studies Depression Scale). Am J Prev Med. 1994;10(2):77-84. https://doi.org/10.1016/S0749-3797(18)30622-6.

54. Stafford L, Judd F, Gibson P, Komiti A, Quinn M, Mann GB. Comparison of the hospital anxiety and depression scale and the center for epidemiological studies depression scale for detecting depression in women with breast or gynecologic cancer. Gen Hosp Psychiatry. 2014;36(1): 74-80. https://doi.org/10.1016/j.genhosppsych.2013.08.010.

55. Fraser L, Burnell M, Salter LC, Fourkala E-O, Kalsi J, Ryan A, Gessler S, Gidron $Y$, Steptoe A, Menon U: Identifying hopelessness in population research: a validation study of two brief measures of hopelessness. BMJ Open. 2014; 4(5):e005093. https://doi.org/10.1136/bmjopen-2014-005093.

56. Schalet BD, Pilkonis PA, Yu L, Dodds N, Johnston KL, Yount S, et al. Clinical validity of PROMIS depression, anxiety, and anger across diverse clinical samples. J Clin Epidemiol. 2016;73:119-27. https://doi.org/10.1016/j.jclinepi.2 015.08.036.

57. Thewes B, Butow P, Zachariae R, Christensen S, Simard S, Gotay C. Fear of cancer recurrence: a systematic literature review of self-report measures. Psycho-oncology. 2012;21(6):571-87. https://doi.org/10.1002/pon.2070.

58. Gross JJ, John OP. Individual differences in two emotion regulation processes: implications for affect, relationships, and well-being. J Pers Soc Psychol. 2003;85(2):348-62. https://doi.org/10.1037/0022-3514.85.2.348.

59. Abler B, Kessler H. Emotion Regulation Questionnaire - Eine deutschsprachige Fassung des ERQ von Gross und John. Diagnostica. 2009; 55(3):144-52. https://doi.org/10.1026/0012-1924.55.3.144.

60. Yu L, Buysse DJ, Germain A, Moul DE, Stover A, Dodds NE, et al. Development of short forms from the PROMIS sleep disturbance and sleeprelated impairment item banks. Behav Sleep Med. 2011;10(1):6-24. https:// doi.org/10.1080/15402002.2012.636266.

61. Bredle JM, Salsman JM, Debb SM, Arnold BJ, Cella D. Spiritual well-being as a component of health-related quality of life: the functional assessment of chronic illness therapy — spiritual well-being scale (FACIT-Sp). Religions. 2011;2(1):77-94. https://doi.org/10.3390/rel2010077.

62. Schulz U, Schwarzer R. Social support in coping with illness: the Berlin social support scales (BSSS). Diagnostica. 2003;49(2):73-82. https://doi.org/10.102 6//0012-1924.49.2.73

63. Craig $C L$, Marshall AL, Sjostrom M, Bauman AE, Booth ML, Ainsworth BE, et al. International physical activity questionnaire: 12-country reliability and validity. Med Sci Sports Exerc. 2003;35(8):1381-95. https://doi.org/10.1249/01. MSS.0000078924.61453.FB.

64. Schroder H, Fito M, Estruch R, Martinez-Gonzalez MA, Corella D, SalasSalvado J, et al. A short screener is valid for assessing Mediterranean diet 
adherence among older Spanish men and women. J Nutr. 2011;141(6): 1140-5. https://doi.org/10.3945/jn.110.135566.

65. Quandt SA, Verhoef MJ, Arcury TA, Lewith GT, Steinsbekk A, Kristoffersen AE, et al. Development of an international questionnaire to measure use of complementary and alternative medicine (I-CAM-Q). J Altern Complement Med. 2009;15(4):331-9. https://doi.org/10.1089/acm.2008.0521.

66. Quandt SA, Ip EH, Saldana S, Arcury TA. Comparing two questionnaires for eliciting CAM use in a multi-ethnic US population of older adults. Eur J Integr Medicine. 2012;4(2):e205-11. https://doi.org/10.1016/j.eujim.2 011.12.009.

67. Lo Re ML, Schmidt S, Güthlin C. Translation and adaptation of an international questionnaire to measure usage of complementary and alternative medicine (I-CAM-G). BMC Complement Altern Med. 2012;12:259. https://doi.org/10.1186/1472-6882-12-259.

68. Chang VT, Hwang SS, Feuerman M, Kasimis BS, Thaler HT. The memorial symptom assessment scale short form (MSAS-SF). Cancer. 2000;89(5): 1162-71. https://doi.org/10.1002/1097-0142(20000901)89:5<1162::AIDCNCR26>3.0.CO;2-Y.

69. Attkisson CC, Zwick R. The client satisfaction questionnaire. Psychometric properties and correlations with service utilization and psychotherapy outcome. Eval program plann. 1982;5(3):233-7. https://doi.org/10.1016/01497189(82)90074-X.

70. Ostermann T, Ehlers J, Warzecha M, Hohenberg G, Zupanic M. Development of an online-system for assessing the progress of knowledge acquisition in psychology students. Data 2018: Proceedings of the 7th International Conference on Data Science, Technology and Applications: 77-82. https:// doi.org/10.5220/0006892500770082.

71. Peduzzi P, Concato J, Kemper E, Holford TR, Feinstein AR. A simulation study of the number of events per variable in logistic regression analysis. J Clin Epidemiol. 1996;49(12):1373-9. https://doi.org/10.1016/ S0895-4356(96)00236-3.

72. Vittinghoff $E$, McCulloch CE. Relaxing the rule of ten events per variable in logistic and cox regression. Am J Epidemiol. 2007;165(6):710-8. https://doi. org/10.1093/aje/kwk052.

73. Floden $\mathrm{L}$, Bell ML. Imputation strategies when a continuous outcome is to be dichotomized for responder analysis: a simulation study. BMC Med Res Methodol. 2019;19(1):161. https://doi.org/10.1186/s12874-019-0793-x.

74. Schomaker M, Heumann C. Model selection and model averaging after multiple imputation. Comput Stat Data An. 2014;71:758-70. https://doi.org/1 0.1016/j.csda.2013.02.017.

75. Rethorst CD, South CC, Rush AJ, Greer TL, Trivedi MH. Prediction of treatment outcomes to exercise in patients with nonremitted major depressive disorder. Depress Anxiety. 2017;34(12):1116-22. https://doi.org/1 $0.1002 / d a .22670$

76. Tibshirani R. Regression shrinkage and selection via the Lasso. J R Stat Soc B. 1996;58(1):267-88

77. Friedman J, Hastie T, Tibshirani R: Regularization Paths for Generalized Linear Models via Coordinate Descent. J Stat Softw. 2010;33(1):1-22.

78. Breiman L. Random forests. Mach Learn. 2001;45(1):5-32. https://doi.org/10.1 023/A:1010933404324.

79. Bharathidason S, Venkateswaran J. Improving classification accuracy based on random Forest model with uncorrelated high performing trees. Int J Comput Appl. 2014;101(13):26-30. https://doi.org/10.5120/17749-8829.

80. Fouladbakhsh JM, Stommel M. Gender, symptom experience, and use of complementary and alternative medicine practices among cancer survivors in the U.S. cancer population. Oncol Nurs Forum. 2010;37(1):E7-E15. https:// doi.org/10.1188/10.ONF.E7-E15.

\section{Publisher's Note}

Springer Nature remains neutral with regard to jurisdictional claims in published maps and institutional affiliations.

Ready to submit your research? Choose BMC and benefit from:

- fast, convenient online submission

- thorough peer review by experienced researchers in your field

- rapid publication on acceptance

- support for research data, including large and complex data types

- gold Open Access which fosters wider collaboration and increased citations

- maximum visibility for your research: over $100 \mathrm{M}$ website views per year

At BMC, research is always in progress.

Learn more biomedcentral.com/submissions 